\title{
Filter Banks for Cyclic-prefixing the Nonuniform DMT System
}

\author{
P. P. Vaidyanathan and Bojan Vrcelj \\ Dept. Electrical Engr., Caltech. Pasadena, CA \\ ppvnath@systems.caltech.edu bojan@systems.caltech.edu
}

\begin{abstract}
The cyclic prefix system is used in discrete multitone channels for frequency domain equalization. This is based on the inversion of samples of the channel frequency response at uniformly spaced points (DFT coefficients). In this paper we consider nonuniformly spaced samples of the channel frequency response, especially ocatve-spaced. The anticipated advantage is that for channels with rapidly decaying frequency responses, therc are relatively fewer equalizer coefficients with unduly large values, and this helps to reduce amplification of channel noise at the receiver. We show how to combine a wavelet-like filter bank with traditional DFTs to achieve this goal. While the idea appears to be exciting and opens up interesting problems, its merits still remain to be evaluated. ${ }^{1}$
\end{abstract}

\section{INTRODUCTION}

Discrete multitone modulation, which today is used in DSL technology, employs the cyclic prefix system for frequency domain equalization [7]. The introduction of cyclic-prefix with length equal to the order of the (FIR) channel $C(z)$ ensures that the channel can be equalized by using the inverse of $C\left(e^{j \omega}\right)$ at uniformly sampled set of frequencies $\omega_{k}=2 \pi k / M$ (Fig. 1(a)) where $M$ is the block length used in the cyclic prefixing [6]. It is well known that when the samples $C\left(e^{j \omega_{k}}\right)$ get very small, the frequency domain equalizers $1 / C\left(e^{j \omega_{k}}\right)$ auplify channel noise severely. If the samples can be crowded more effectively near the regions where $\left|C\left(e^{j \omega}\right)\right|$ is large as in Fig. 1(b), this problem can be reduced significantly. In this paper we develop structures which allow us to obtain such nonuniform samples by using a combination of traditional uniform DMT systerns and nonuniform filter banks. While the idea is quite interesting, further work is required to examine its practical merits. There are a few open problems that still need to be addressed.

Preliminaries. Standard multirate notations (decimators, expanders, delay chains, and so forth) will be used throughout, and can be found, for example, in [8]. Thus $\downarrow M$ and $\uparrow M$ represent the $M$-fold decimator and expander respectively, and $[X(z)]_{\perp M}$ represents the $z$ transform of the decimated version $x(M n)$. The blocked version $\mathbf{a}(n)$ of a signal $a(n)$ with block length $K$ is defined as shown in Fig. 2, and Fig. 3 shows the unblocking operation. It will be assumed throughout that the channel is an

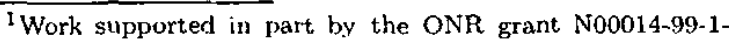
1002, USA.
}

FIR filter

$$
C(z)=\sum_{n=0}^{L} c(n) z^{-n}
$$

followed by additive noise $e(n)$ (Fig. 4). The FIR assumption is usually justified because of the channel shortening (or time-domain equalization) step that normally precedes cyclic prefixing [4].

(a)

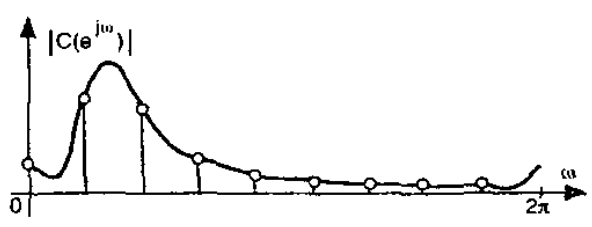

(b)

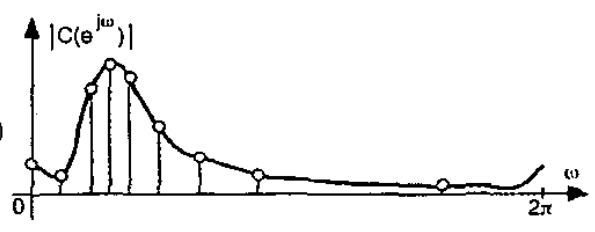

Fig. 1. Sampling the channel frequency response for equalization. (a) Uniform sampling, and (b) nonuniform sampling.

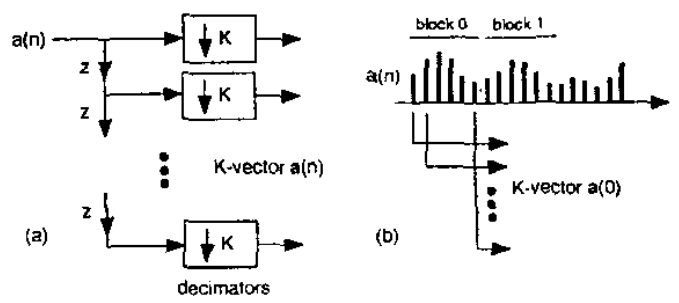

Fig. 2. (a) The blocking schomatic, and (b) an example. 


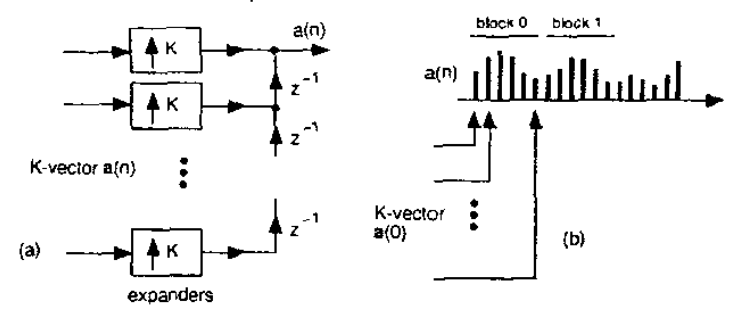

Fig. 3. (a) The unblocking schematic, and (b) an example.

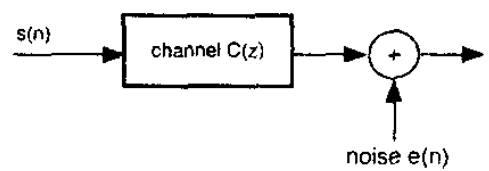

Fig. 4. A linear channel with additive noise.

\section{THE TRADITIONAL CYCLIC PREFIX SYSTEM}

Fig. 5(a) shows how a vector signal $\mathbf{s}(n)$ is cyclic-prefixed. Here $\mathbf{s}(n)$ is an $M$-component vector

$$
\mathbf{s}(n)=\left[\begin{array}{c}
s_{0}(n) \\
s_{1}(n) \\
\vdots \\
s_{M-1}(n)
\end{array}\right]
$$

and $\mathbf{p}(n)$ is a copy of the last $L$ components of $\mathrm{s}(n)$, that is,

$$
\mathbf{p}(n)=\left[\begin{array}{c}
s_{M-L}(n) \\
\vdots \\
s_{M \cdots 1}(n)
\end{array}\right]
$$

The $(M+L)$-vector

$$
\mathbf{x}(n) \triangleq\left[\begin{array}{l}
\mathrm{p}(n) \\
\mathrm{s}(n)
\end{array}\right]
$$

is said to be the $(L, M)$ cyclic prefixed version of $\mathbf{s}(n)$. This is then unblocked to obtain the scalar signal $x(n)$, which is transmitted on the channel. The received signal $y(\dot{n})$ is blocked with block length $L+M$, and the first $L$ components of each block deleted. The resulting $M$-vector $\mathbf{y}(n)$ is the received version of the transmitted vector $s(n)$. The usefulness of cyclic prefix systems arises when the channel can be assumed to be FIR with order $L<M$. In this case the transformation from $s(n)$ to $y(n)$ is the simple system shown shcematically in Fig. 5(b), where $\mathrm{C}$ is a $M \times M$ circulant matrix, and $\mathrm{e}(n)$ is the $M$-blocked version of the channel noise $e(n)$. For example, when $M=6$ and $L=3$ we have

$$
\mathbf{C}=\left[\begin{array}{cccccc}
c(0) & 0 & 0 & c(3) & c(2) & c(1) \\
c(1) & c(0) & 0 & 0 & c(3) & c(2) \\
c(2) & c(1) & c(0) & 0 & 0 & c(3) \\
c(3) & c(2) & c(1) & c(0) & 0 & 0 \\
0 & c(3) & c(2) & c(1) & c(0) & 0 \\
0 & 0 & c(3) & c(2) & c(1) & c(0)
\end{array}\right]
$$

The fact that $\mathbf{C}$ is circulant implies that it can be diagonalized by the DFT' nnatrix [5]. More precisely

$$
\mathbf{C}=\mathbf{W}^{-1} \boldsymbol{\Lambda}_{c} \mathbf{W}
$$

where $\mathrm{W}$ is the $M \times M \mathrm{DFT}$ matrix with elements

$$
[\mathbf{W}]_{k m}=W^{k m}
$$

with $W=e^{-2 \pi j / M}$. Here

$$
\boldsymbol{\Lambda}_{c}=\operatorname{diag}\{C[0], C[1], \ldots C[M-1]\}
$$

where $C[k]$ are the DFT coefficients of the channel, i.e.,

$$
C[k]=\sum_{n=0}^{M-1} c(n) W^{i k n}=\sum_{n=0}^{L} c(n) e^{-j 2 \pi k n / M}
$$

Evidently $C[k]$ are samples of the channel resposne $C\left(e^{j \omega}\right)$ at the uniformly spaced DFT frequencies $\omega_{k}=2 \pi k / M$. If these are nonzero for all $M$ values of $k$, then $\mathbf{C}$ is nonsingular, and we can equalize the effect of the channel. In practice the equalization is done by inserting the DFT and IDF' matrices as shown in Fig. 6. The signal component $\mathbf{s}(n)$ sees the cascade of $\mathbf{W}^{-1}, \mathbf{C}$, and $\mathbf{W}$ which is equal to $\boldsymbol{\Lambda}_{c}$. This is cancelled by the equalizer $\boldsymbol{\Lambda}_{c}^{-1}$. If the channel frequency response $C\left(e^{j \omega}\right)$ is close to zero near any of the DF' $\mathrm{D}$ frequencies, then $C[k]$ is very small there. This means that $\Lambda_{c}^{-1}$ has large numbers in it, and there could be severe noise amplification.

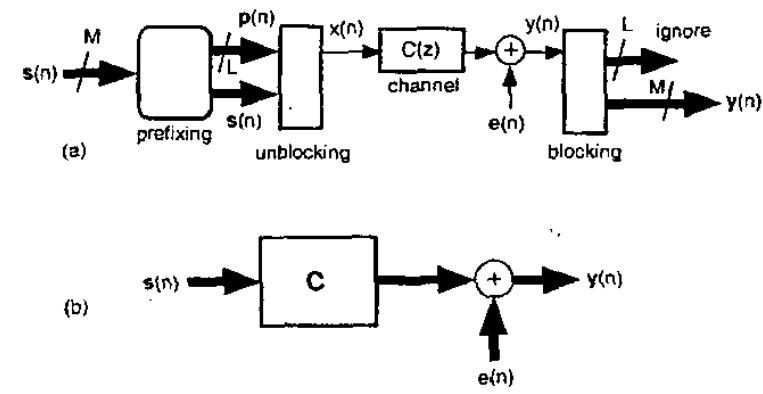

Fig. 5. (a) The basic idea of the $(L, M)$ cyclic prefix system, and (b) equivalent schematic. 


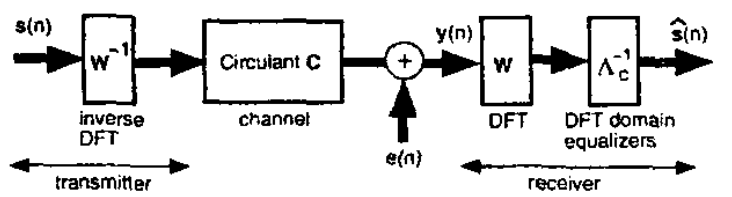

Fig. 6. The cyclic prefix transceiver.

The cyclic prefix system just described will be denoted as the $(L, M)$ cyclic prefix, to remind ourself that the two integers $L$ (prefix length) and $M$ (original block length) are involved in the definition. In practical applications the vector signal $\mathbf{s}(n)$ is typically derived from a scalar symbol stream in one of two possible ways.

(a)

$s(n)=0000000000000000000000000000000000000$ $\underset{\text { B-bit block }}{\rightarrow} \rightarrow \underset{\text { B-bit block }}{\rightarrow} \rightarrow$

(b)
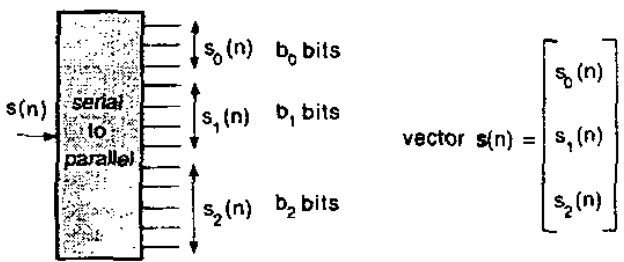

Fig. 7. The $(B, M)$ parser. (a) Parsing a binary sequence $s(n)$ into $M$ groups, $B$ bits at a time, and (b) schematic block diagram.

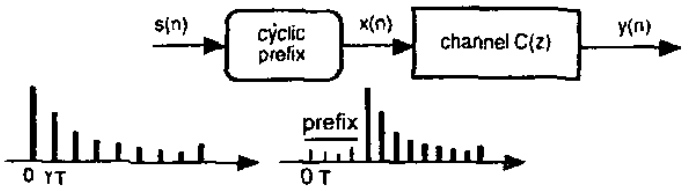

Fig. 8. Schematic of bandwidth expansion.

1. Parsing. This is the most common situation. Here a scalar binary symbol stream $s(n)$ is divided into $B$ bit blocks, and each block divided into $M$ groups as demonstrated in Fig. 7 (a) for $M=3$. The $k$ th group then defines a $b_{k}$-bit symbol stream $s_{k}(n)$ (possibly converted to a PAM or QAM format) which forms the $k$ th component of $\mathbf{s}(n)$, thereby defining $\mathbf{s}(n)$ completely (Fig. $7(\mathrm{~b})$ ). We shall refer to the parser as the $(B, M)$ parser ( $B$ bits turned into $M$ symbol streams). The inverse system at the receiver which turns the $M$ symbol streams $s_{k}(n)$ back into the binary stream $s(n)$ is called the $(B, M)$ unparser.

2. Blocking. In some applications $\mathbf{s}(n)$ is a vector obtained by blocking a scalar symbol st ream $s(n)$, possibly a PAM or QAM stream (Fig. 2).
Since there are $M+L$ samples in the same duration that was originally occupied by $M$ samples, there is a bandwidth expansion ratio $\gamma=(M+L) / M$ which can be regarded as the price paid for the cyclic prefixing. This means that the symbol spacing at the channel input is closer than the original spacing, as schematized in Fig. 8. Extensions of the cyclic prefix technique in fractionally spaced equalization, and in blind equalization methods are described in $[9]$ and $[10]$.

\section{NONUNIFORM DMT SYSTEMS}

We explain the idea of nonuniform DMT with an example, which is readily generalized. The first step in the development is to split the original binary symbol stream into substreams with different symbol spacings.

(a)
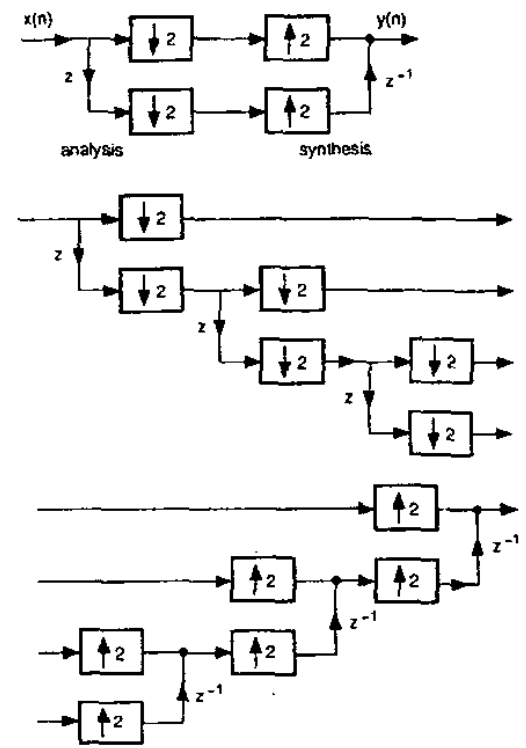

Fig. 9. The nonuniform delay chain system. (a) Two channel perfect reconstruction system, (b) analysis tree, and (c) synthesis tree.

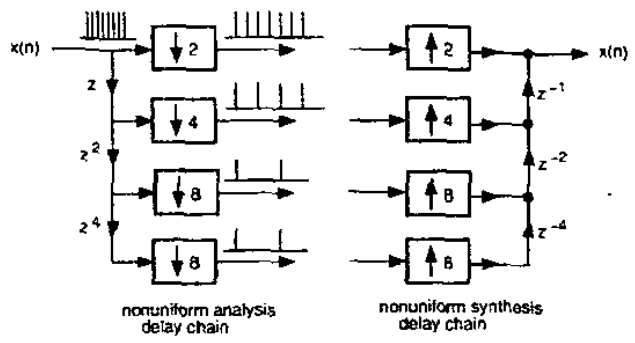

Fig. 10. Equivalent nonuniform analysis/synthesis chain.

To accomplish this, consider Fig. 9(a) which shows a twochannel delay chain filter bank [8] with the perfect reconstruction properly $y(n)=x(n)$. Suppose the two-channel analysis banks are connected in a tree to obtain the system 
of Fig. 9(b), and similarly, synthesis banks connected as in Fig. 9(c). This analysis/synthesis system is equivalent to the nonuniform perfect reconstruction system shown in Fig. 10. In this system, the input stream $x(n)$ is merely partitioned into substreams of different rates, as demonstrated in the figure. The synthesis system simply interleaves them appropriately to obtain $x(n)$ again.
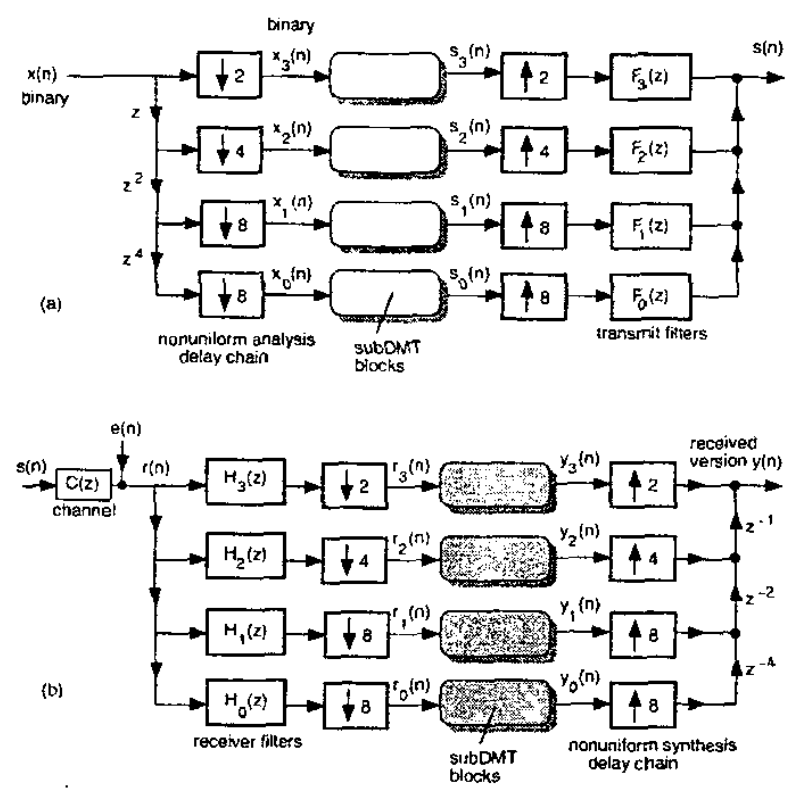

Fig. 11. The nonuniform DMT filter bank. (a) Transmitter sidc, and (b) receiver side.

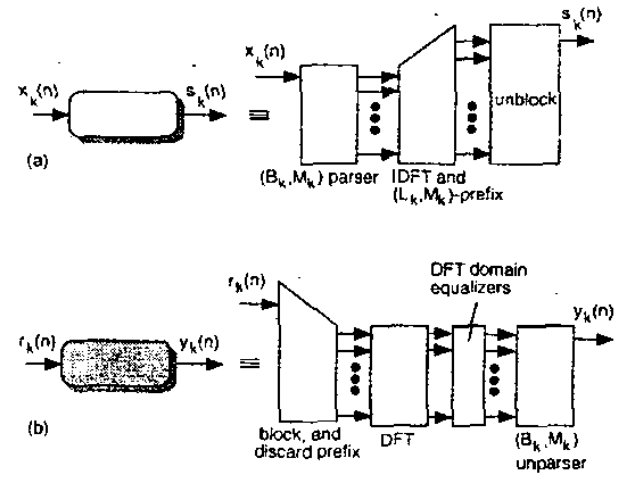

Fig. 12. The subDMT systems in the nonuniform DMT filter bank. (a) Transmitter side and (b) receiver side.

Now consider Fig. 11(a). This is again an exarnple with four channeis, and demonstrates the main features of the system. The reciprocals of the decimation ratios $(2,4,8,8)$ add up to unity. The input binary stream is first partitioned nonuniformly, and then the substreams are processed by subDMT blocks. Each subDMT block performs the operation described in Fig. 12(a) for the kth channel.
Thus, there is $\left(B_{k}, M_{k}\right)$ parsing of the signal $x_{k}(n)$, followed by IDFT and then $\left(L_{k}, M_{k}\right)$ cyclic prefixing. The resulting vector is then unblocked to obtain $s_{k}(n)$. The subDMT blocks create bandwidth expansion by a factor

$$
\gamma_{k}=\left(L_{k}+M_{k}\right) / M_{k}
$$

Even though the quantities $B_{k}, M_{k}$, and $L_{k}$ need not be the same for all the subDMT blocks, we will assume that $\gamma_{k}$ is the same for all $k$. This ensures that the inputs to the transmitter filters $F_{k}(z)$ in Fig. 11(a) have identical symbol spacing for all $k$. The filter outputs can therefore be added to get the signal $s(n)$ which is eventually transmitted on the channel.

The receiver for the nonuniform DMT system is shown in Fig. 11(b). Notice that the set of filters $\left\{F_{k}(z), H_{k}(z)\right\}$ acts as a (nonuniform) transmultiplexer for the channel as shown separately in Fig. 13. The channel output $r(n)$ is first separated by the receiver filters $H_{k}(z)$ into subbands $r_{k}(n)$. Fach of the subband signals then goes through the receiver counterpart of the subDMT' system, as described in Fig. 12(b): the cyclic prefix is first removed, then a DFT performed, and finally the unparser generates the received version $y_{k}(n)$ of the symbol stream $x_{k}(n)$. Evidently $y_{k}(n)$ has different spacing for different $k$. Interleaving these using the nonuniform delay chain, we finally obtain $y(n)$ which is the received version of $x(n)$. Needless to say, if the input $x(n)$ in Fig. $11(a)$ is not binary but an arbitrary signal, we can replace the parser and unparser in Fig. 12 by blocking and unblocking operations.

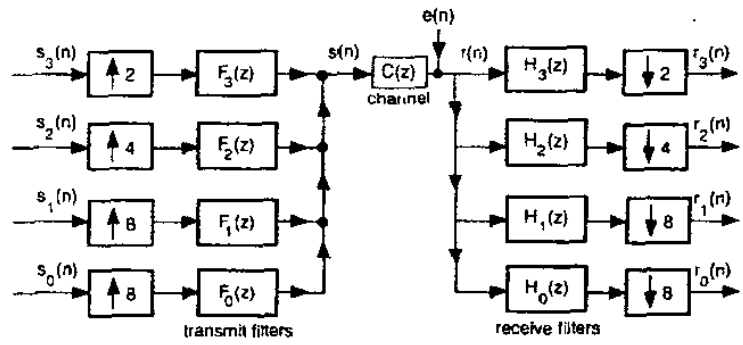

Fig. 13. The nonuniform transmultiplexer.

\section{OPERATION WITH IDEAL FILTERS}

Continuing our example of the four channel nonuniform system in Fig. 11, let us assume that the filters $F_{k}(z)$ are ideal with responses as in Fig. 14(a). Assuming $H_{k}(z)$ have identical responses, it follows that $F_{k}\left(e^{j \omega}\right) H_{m}\left(e^{j \omega}\right)=$ 0 for $k \neq m$ so that there is no crosstalk between subchannels of the transmultiplexer. The system of Fig. 13 therefore merely splits the channel into four subchannels with unequal bandwidths. The path from $s_{k}(n)$ to $r_{k}(n)$ is the filter

$$
C_{k}(z)=\left[H_{k}\left(e^{j \omega}\right) F_{k}\left(e^{j \omega}\right) C\left(e^{j \omega}\right)\right]_{1 n_{k}}
$$

where $\downarrow n_{k}$ is the decimator in the $k$ th branch. For example, the quantity $H_{1}\left(e^{j \omega}\right) F_{1}\left(e^{j \omega}\right) C\left(e^{j \omega}\right)$ is the piece of the channel highlighted in Fig. 14(b) and the 8-fold decimation 
yields Fig. 14(c). Thus the ideal nonuniform transmultiplexer merely separates different portions of the channel and spreads them out into the region $[0,2 \pi\}$. The subDMT systems perform equalization by sampling the subchannel frequency responses as shown by the vertical lines in Fig. 14(c). Thus, the set of samples of the channel created by all the different paths appears nonuniform as demonstrated in Fig. 14(d) which assumes DMT block length $M=4$ for simplicity (in practice this could be different from the number of transmultiplexer channels).

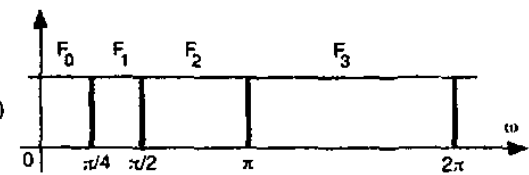

(b)

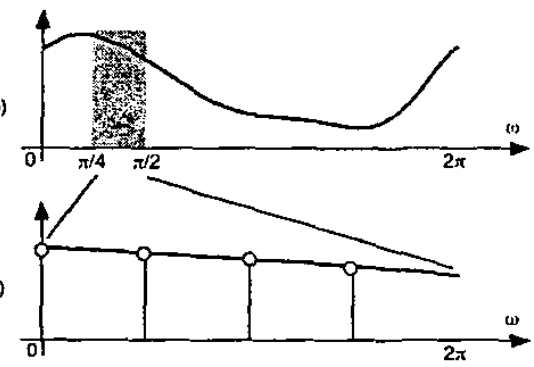

(d)

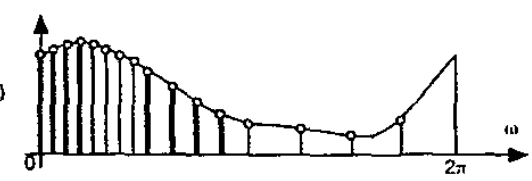

Fig. 14. (a) Example of ideal filter responses, (b) portion of channel $C\left(e^{j \omega}\right)$ in the band of $F_{1}(z)$, (c) decimation by 8 , and (d) nonuniform sampling of the channel effected by the nonuniform DMT system.

We can realize the nonuniform transmultiplexer by building a tree structured filter bank with two-channel modules. Assume for example that a two-channel filter bank costs $N$ multipliers per symbol. Then because of the decimation involved, the total cost of the tree structure is $N+0.5 N+0.25 N \ldots \approx 2 N$. With filters of moderate or der, e.g., $N=51$, we can get excellent approximations of the ideal response, and cross talks created by the transmultiplexer can be ignored. In practice the subDMT system can atso choose not to allocate bits at the band edges where the effects of cross talk are most severe. With cross talks eliminated or ignored, the system from $s_{k}(n)$ to $r_{k}(n)$ in Fig. 11 has the transfer function $C_{k}(z)$ given in $\mathrm{Eq}$. (1). This can be regarded as the effective channel to be equalized by the $k$ th subDMT system. Note that the cost of the filters $\left\{F_{k}(z), H_{k}(z)\right\}$ is typically negligible compared to the costs of the DFT matrices involved in the subDMT systems. For example if $M=256$ in a subDMT system, this costs about $256 * 8 / 2=1,024$ multiplications at the decimated rate. Assuming that all the DMT systems operating at the decimated rate have the same DFT size, the total cost of DMT is 1,024 multiplications at the original rate (i.e., per sample of $x(n)$ in Fig. 11).

\section{CONCLUDING REMARKS}

While we have used the four-channel nonuniform system as an example throughout, it is possible to get nearly arbitrary tilings of the frequency axis by using nonbinary treestructured filter banks (wavelet packets). As the reader can see, the purpose of the paper has mainly been to introduce the idea rather than delve into the details of many of the open problems that remain to be addressed. We know that Eq. (1) faithfully represents the transfer function of the $k$ th subchannel. But equally important are the cross talk terms between the channels, which have the form

$$
C_{k m n}(z)=\left[H_{k}\left(e^{j \omega}\right) F_{m}\left(e^{j \omega}\right) C\left(e^{j \omega}\right)\right]_{\left\{g_{k m}\right.}
$$

where $g_{k m 1}=\operatorname{gcd}\left(n_{k}, n_{m}\right)$. Designing the filters to satisfy the biorthogonality condition $\left[H_{k} F_{m}\right]_{\downarrow g_{k m}}=0$ for $k \neq$ $m$ does not ensure $C_{k m}(z)=0$. Even when the filters $F_{m}(z)$ and $H_{k}(z)$ have very sharp cutoffs, there is always a transition region of overlap which creates interchannel interference and increases error probability. Finally, even though we assign fewer samples to bands of lower energy (e.g., the band $[\pi, 2 \pi]$ in Fig. 14(d)), this channel also has the largest number of symbols per second (because the decimator and expander are only two-fold, see Fig. 13). This will offset, to some extent, the advantage obtained by allocating fewer frequency samples in that band! Further work needs to be done to take into account these issues, before the nonuniform cyclic prefix is deemed a practical alternative.

\section{REFERENCES}

[1] Benyassine, A., and Akansu, A. N., "Performance analysis and optimal structuring of subchannels for discrete multitone transceivers," Proc. ISCAS, 1995.

[2] Bingham, J. A. C. "Multicarrier modulation for data transmission: an idea whose time has come," IEEE Comm. Mag., pp. 5-14, May 1990.

(3) Kalet, I. "The multitone channel", IEEE Trans. Comm., pp. 119-124, Feb. 1989.

[4] Melsa, J. W., Younce, R. C., Rohrs, C. E., "Impulse response shortening for discrete multitone receivers, "IEEE Trans. Comm, Dec. 1996.

[5] A. Papoulis, Signal analysis, McGraw Hill, 1977

[6] Peled, A., and Ruiz, A. "Frequency domain data transmission using reduced computational complexity algorithms," IEEE ICASSP, pp. 964-967, Denver, CO, Apr. 1980.

(7) Starr, T, Cioff, J. M., and Silverman, P. J. Understanding DSL technology, Prentice Hall, Inc., 1999.

[8] P. P. Vaidyanathan, Multirate systems and filter banks, Prentice Hall, Inc., 1993.

[9] P. P. Vaidyanathan and B. Vrcelj, "Fast and robust blind-equalization based on cyclic prefix,"Proc. ICC, New York, Apr-May 2002.

[10] P. P. Vaidyanathan and B. Vrcelj, "Thcory of fractionally spaced cyclic-prefix equalizers,"Proc. ICASSP, Orlando, FL, May 2002. 